\title{
Short-Time Relaxational Dynamics of the "Strong" Glass-Former Methanol
}

\author{
K. L. Ngai* and C. M. Roland* \\ Naval Research Laboratory, Washington, D.C. 20375-5320 \\ Received: October 28, 1996; In Final Form: February 12, $1997^{\otimes}$
}

\begin{abstract}
According to the coupling model of relaxation, structural relaxation in glass-forming liquids is comprised of an intermolecularly uncorrelated step ("fast $\alpha$-relaxation process") in the picosecond time range followed by a slowed, intermolecularly cooperative, "slow $\alpha$ " process. Molecular dynamics simulation data [Sindzingre, P.; Klein, M. J. Chem. Phys. 1992, 96, 4681] have shown that for the "strong" liquid methanol, the fast relaxation step is absent. This finding is in contrast to the prominent fast relaxation appearing in fragile liquids about the glass transition temperature. The differing behavior of methanol can be accounted for from an analysis of the self part of the intermediate scattering function, $F_{\mathrm{S}}(k, t)$ according to the coupling model. The latter relates the magnitude of the fast $\alpha$-relaxation to the relaxation time, $\tau^{*}$, and to the exponent $\beta$ of the slow $\alpha$-process described by the stretched exponential function $\exp \left[-\left(t / \tau^{*}\right)^{\beta}\right]$. This function fits the experimental $F_{\mathrm{S}}(k, t)$ for $t$ longer than 2 ps. The apparent absence of a fast relaxation step in methanol is shown to be a consequence of the weak intermolecular constraints governing the dynamics in "strong" liquids, a result consistent with the prominence of the fast process in polymers and other fragile glass-formers. This conclusion is supported by dielectric relaxation data (frequencies up to $90 \mathrm{GHz}$ ) and far-infrared data (>150 $\mathrm{GHz}$ ) on methanol.
\end{abstract}

\section{Introduction}

Short-time relaxational processes in glass-forming-liquids have been the subject of intense research, using various techniques including quasielastic neutron scattering, ${ }^{1-6}$ dynamic light scattering, ${ }^{7-9}$ and molecular dynamics simulations. ${ }^{10-12}$ Much of this activity has been stimulated by mode coupling theory (MCT) ${ }^{13}$ for example, in assessing the theory's prediction of a fast (ca. picosecond) $\beta$-process. The latter is not a relaxation in the conventional sense but refers to a putative susceptibility minimum lying at frequencies between a temperature-insensitive microscopic peak, associated with vibrational motions, and the primary $\alpha$-relaxation. Evidence for the existence of a fast process has indeed been found in some polymers, ${ }^{4,5,14}$ as well as several small molecule glass-formers such as $0.6 \mathrm{Ca}\left(\mathrm{NO}_{3}\right)_{2}-0.4 \mathrm{KNO}_{3}(\mathrm{CKN})^{1,2,7}$ and $o$-terphenyl (OTP). ${ }^{3}$ These fast processes have not always been identified as the $\beta$-process of MCT but rather as another possiblity as described below. ${ }^{4,5,15-18}$ The materials exhibiting a fast process are all considered "fragile" glass-formers ${ }^{1-14}$ (i.e., exhibiting relaxation times that decrease markedly with a change in $T_{\mathrm{g}}$ normalized temperature ${ }^{19}$ ). The expectation from MCT is that all glass-formers, "fragile" or "strong" (both terms referring to the temperature sensitivity of the relaxation times), will exhibit a fast $\beta$-process.

Especially for polymers, ${ }^{4,5,14}$ experimental data often depart substantially from MCT, leading some experimentalists ${ }^{4,5}$ to consider an alternative, the coupling model (CM) ${ }^{15-18,20}$ for the interpretation of their data. The physical basis of the model can be found in ref 20 and will not be reiterated here. The CM predicts the existence of a fast $\alpha$-relaxation process, which has no relationship to the MCT fast $\beta$-process, although they contribute at similar frequencies. In the $\mathrm{CM}$, this fast $\alpha$-relaxation process represents the initial phase of structural relaxation, occurring prior to the onset of intermolecular cooperativity effects. The properties of the CM's fast $\alpha$-process are in agreement with the neutron scattering data of polymers and

\footnotetext{
${ }^{\otimes}$ Abstract published in Advance ACS Abstracts, May 1, 1997.
}

dielectric relaxation data of $\mathrm{CKN} .{ }^{18}$ Hence, there are at least two possible interpretations of experimental data for those glassformers that exhibit a prominent fast process - an MCT fast $\beta$ process and the fast $\alpha$-relaxation of the CM. ${ }^{15-18}$

More recently, experimental studies have been carried out of the short-time dynamics in nonfragile glass-formers, such as $\mathrm{ZnCl}_{2},{ }^{21} \mathrm{~B}_{2} \mathrm{O}_{3}$, ${ }^{22}$ and glycerol. ${ }^{6,8}$ In these "intermediate liquids", no prominent fast process is observed, notwithstanding the MCT prediction of its presence in both fragile and nonfragile liquids. To reconcile this deviation from MCT, it has been suggested that masking by a dominant boson peak causes the apparent absence of a fast $\beta$-process in nonfragile liquids. ${ }^{21}$ Such a possibility has been considered ${ }^{21}$ for $\mathrm{ZnCl}_{2}$ as studied by lightscattering experiments, although the possibility remains of simply the absence of any fast process. ${ }^{20}$ More problematic, however, is the case of glycerol. ${ }^{6}$ Neutron scattering measurements on glycerol showed that the crossover from vibrations to relaxations occurs much more quickly than for other molecular liquids. The fast $\beta$-relaxation region of MCT in glycerol, therefore, would have to be rather small, if it exists at all. ${ }^{6,17}$

The prominence of the fast $\alpha$-process in fragile liquids, and its lack thereof in nonfragile liquids, can be predicted from the $\mathrm{CM}$, according to which the fast $\alpha$-process is given as

$$
C_{\text {rel }}(t)=\exp \left(-t / \tau_{0}\right) \quad \text { for } t<t_{\mathrm{c}}
$$

where $t_{\mathrm{c}}$ is a crossover time on the order of a picosecond and $\tau_{0}$ is the noncooperative relaxation time. For $t>t_{\mathrm{c}}$, the averaged relaxational dynamics are slowed by intermolecular constraints, giving rise to the slow $\alpha$-process. The correlation function crosses over to the Kohlrausch function:

$$
C_{\text {rel }}(t)=\exp \left[-\left(t / \tau^{*}\right)^{\beta}\right] \text { for } t>t_{\mathrm{c}}
$$

The continuity condition at $t_{\mathrm{c}}, \exp \left(-t_{\mathrm{c}} / \tau_{0}\right)=\exp \left[-\left(t_{\mathrm{c}} / \tau^{*}\right)^{\beta}\right]$, leads to the relation

$$
\tau^{*}=\left[t_{\mathrm{c}}^{-n} \tau_{0}\right]^{1 / \beta}
$$




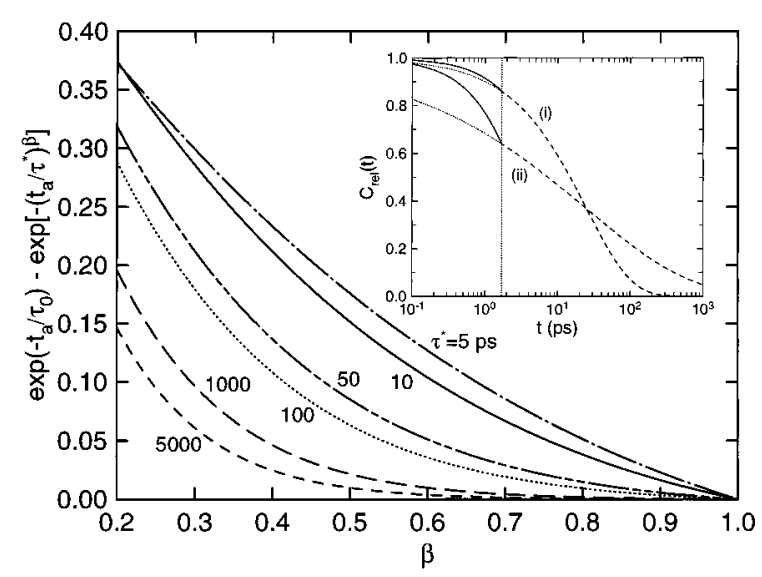

Figure 1. Dependence of the prominence of the fast $\alpha$-relaxation on $\beta$ for various $\tau^{*}$ calculated as $\exp \left(-t_{\mathrm{a}} / \tau_{0}\right)-\exp \left[-\left(t_{\mathrm{a}} / \tau^{*}\right)^{\beta}\right]$, where $t_{\mathrm{a}}$ $=0.5 \mathrm{ps}$. The inset illustrates for the same $\tau^{*}$, (i) a weak, fast $\alpha$-relaxation process for a large $\beta$ value of 0.70 and (ii) a prominent fast $\alpha$-relaxation process for a small $\beta$ value of 0.30 .

Stronger intermolecular constraints make the relaxation more cooperative. The latter is reflected in eq 2 by a smaller value of $\beta$, stretching the relaxation over a broader time range and to longer times. The latter effect is a consequence of eq 3 , which yields a longer $\tau^{*}$ for smaller $\beta$ under the condition that $t_{\mathrm{c}}$ and $\tau_{0}$ are the same and $\tau_{0}>t_{\mathrm{c}}$. Thus, in the context of the coupling model, fragile liquids (smaller $\beta$ ) are more intermolecularly constrained, while strong liquids experience weaker coupling.

The relation between the fast and slow $\alpha$-processes is illustrated in the inset in Figure 1 for $t_{\mathrm{c}}=1.7 \mathrm{ps}, \tau^{*}=25 \mathrm{ps}$, and two choices of $\beta=0.70$ and 0.30 . We have plotted exp$\left[-\left(t / \tau^{*}\right)^{\beta}\right]$ for both $t>t_{\mathrm{c}}$ (dashed curves) and $t<t_{\mathrm{c}}$ dotted curves), although this function is strictly valid only for $t>t_{\mathrm{c}}$. One measure of the prominence of the fast $\alpha$-process is the difference between $\exp \left(-t_{\mathrm{a}} / \tau_{0}\right)$ and $\exp \left[-\left(t_{\mathrm{a}} / \tau^{*}\right)^{\beta}\right]$ at a given time $t_{\mathrm{a}}<t_{\mathrm{c}}$. Obviously, a larger difference indicates a more prominent fast $\alpha$-process, and when $\beta=1$, the difference is identically zero for any $\tau^{*}$. In Figure 1 we plot the quantity $\exp \left(-t_{\mathrm{a}} / \tau_{0}\right)-\exp \left[-\left(t_{\mathrm{a}} / \tau^{*}\right)^{\beta}\right]$ for an arbitrarily chosen $t_{\mathrm{a}}=0.5$ ps $\left(<t_{\mathrm{c}}\right)$ as a function of $\beta$ with $\tau^{*}$ as a parameter. One can see clearly that the prominence of the fast $\alpha$-process, measured by this difference, rapidly decreases with increasing $\beta$. This relationship is a consequence of eqs $1-3$ of the coupling model, and we are aware of no other model that predicts such a trend.

Phenomenologically, and in accord with the CM, more fragile glass-formers are associated with a smaller $\beta .{ }^{19}$ Polymers as a family have the smallest $\beta$, suggesting why the fast $\alpha$-process is so prominent in their neutron scattering spectra. ${ }^{4,5}$ Conversely, "strong" and intermediate (i.e., large $\beta$ ) glass-formers are predicted to have a weak, fast $\alpha$-process. Hence, the fast process is absent in the experimental data of $\mathrm{ZnCl}_{2}, \mathrm{~B}_{2} \mathrm{O}_{3}$, and glycerol.,17 Note that the terms "fragile" and "strong", proposed by Angell ${ }^{19}$ and commonly used in the field, can be misleading when dynamics are discussed in the context of the CM, as herein. "Fragile" in the Angell sense implies a glass-former whose structural relaxation time is a rapid function of the $T_{\mathrm{g}}$ scaled temperature near $T_{\mathrm{g}}$. Conversely, those liquids that have weak $T_{\mathrm{g}}$-scaled temperature dependencies are expected by Angel to have little structural change with an increase of temperature and hence were referred to as "strong". Theoretical ideas concerning the origin of the various $T_{\mathrm{g}}$-scaled temperature dependencies of glass-forming liquids have changed over time. ${ }^{23}$ However, the terms "fragile" and "strong" have become so wellembedded in the literature that their usage continues. The recently found correlation between $\beta$ and the $T_{\mathrm{g}}$-scaled temperature dependence of the structural relaxation time ${ }^{24-27}$ opens

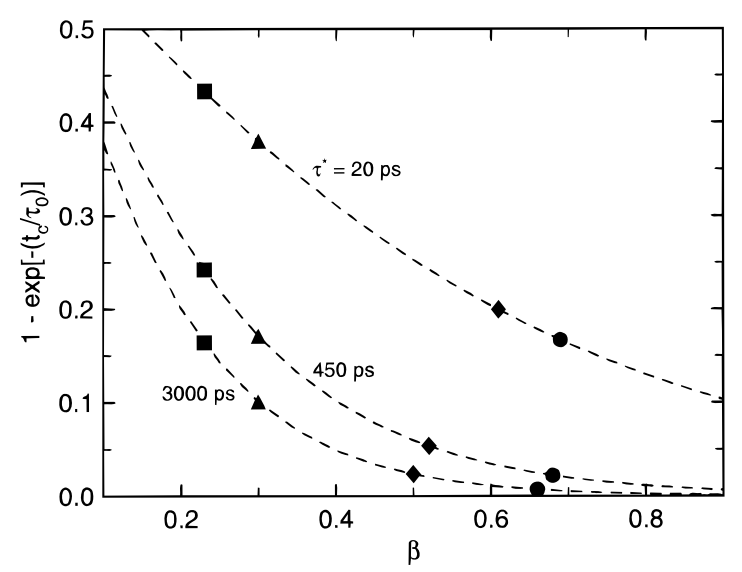

Figure 2. Amount of short-time relaxational decay as a function of the stretch exponent for various $\tau^{*}$, illustrating the connection between fragility and the prominence of the fast process. The points refer to actual (or interpolated) data for poly(vinyl chloride) ( $\mathbf{\square}$ from ref 4), polybutadiene ( $\Delta$ from ref 5), o-terphenyl $(\checkmark$ from ref 17 ), and methanol ( from the lowest three temperatures in Figure 5 below).

up other possibilities concerning the origin of this behavior. Specifically, in the context of the CM, "strong" liquids have weak intermolecular constraints. To avoid this oxymoron, we have suggested the use of "cooperativity" 26 in place of fragility.

Another measure of the fast $\alpha$-prominence is the magnitude of decay, $f_{\mathrm{k}} \equiv 1-\exp \left(-t_{\mathrm{c}} / \tau_{0}\right)$. Figure 2 shows this quantity as a function of $\beta$ for various fixed $\tau^{*}$. For fragile polymers such as polyvinyl chloride ${ }^{4}$ and polybutadiene, ${ }^{5} f_{\mathrm{k}}$ is substantial. Conversely, there is less or even negligible decay of this quantity for intermediate and strong liquids. A survey of the literature indicates that most, if not all, experimental investigations of the short-time dynamics have been carried out on fragile glassformers and the intermediate liquids mentioned above. There is a strong liquid, methanol, that has been studied by molecular dynamics simulation (MDS) by Sindzingre and Klein. ${ }^{12}$ The purpose of the present work is to test our contention that the fast $\alpha$-process is weak in a strong liquid such as methanol.

\section{Results and Discussion}

Sindzingre and Klein (SK) reported MDS for methanol $\left(\mathrm{CH}_{3}-\right.$ $\mathrm{OH}$ ) over a range of temperatures encompassing its glass transition. ${ }^{12}$ The simulated self part of the intermediate scattering function

$$
F_{\mathrm{s}}(k, t)=\left\langle\exp \left(\mathrm{i} k\left[r_{\alpha}(t)-r_{\alpha}(0)\right]\right\rangle\right.
$$

at several temperatures is reproduced in Figure 3. The data represented by symbols in this figure include all that were reported by SK. At low temperatures (below $210 \mathrm{~K}$ ) there is a decay of $F_{\mathrm{s}}(k, t)$ occurring on a time scale of a few tenths of a picosecond (typical of inverse phonon frequencies) with a time dependence that is nearly independent of temperature (see Figure 3). This decay reflects vibrational motions, whose contribution to the relaxation dynamics is not addressed in either the CM or MCT. Since vibrations and relaxations are statistically independent processes, the intermediate scattering function can be expressed as a product of their respective correlation functions. ${ }^{4,5,15-17}$ In the long-time limit (beyond the inverse of the Debye frequency), the vibrational function assumes a constant value, the Debye-Waller factor, $A_{\mathrm{k}}(T)$.

After the initial decay of $F_{\mathrm{s}}(k, t)$ to the value $A_{\mathrm{k}}(T)$, there is no evidence of any fast relaxational process in the picosecond time range. This interesting behavior was pointed out previously by SK in their attempted analysis using MCT. This absence of 


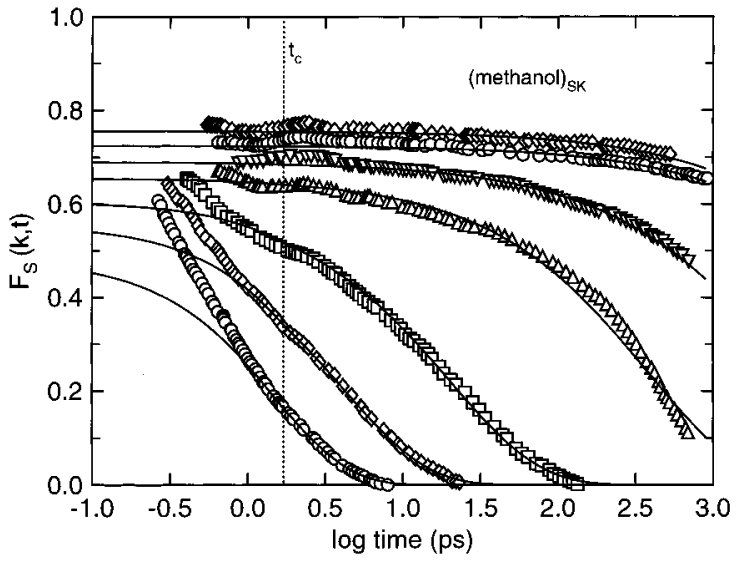

Figure 3. Incoherent structure factor calculated for a $k \approx 2 \AA^{-1}$, using eqs 1 and 2 (solid lines), along with the molecular dynamics simulation results (symbols), taken from ref 12 . From top to bottom, the data correspond respectively to $T=135,155,170,180,210,250$, and 300 $\mathrm{K}$. The crossover time of the coupling model $(=1.7 \mathrm{ps})$ is denoted by the vertical dotted line. Note that although the calculated curves assumed a constant $A_{\mathrm{k}}(T)$, as given in Figure 4, the vibrational contribution actually dominates the response below 2 ps.

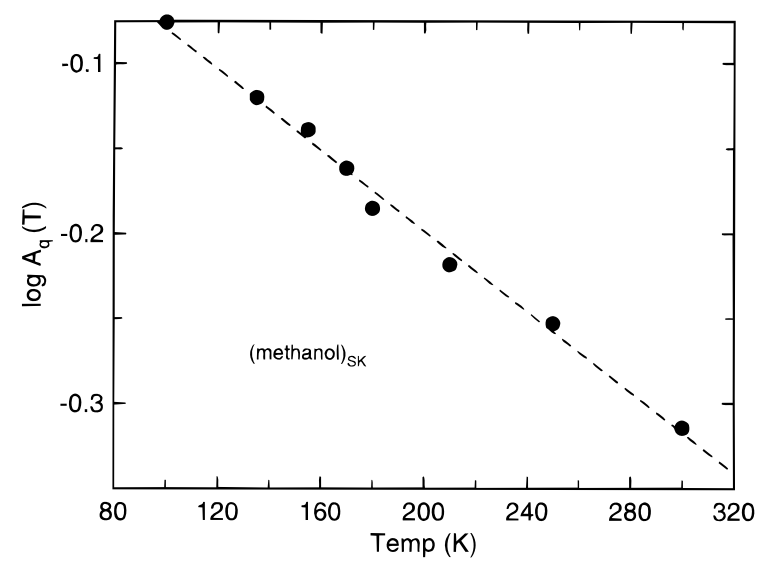

Figure 4. The logarithm of the Debye-Waller factor (i.e., initial plateau value of the incoherent dynamical structure factor prior to the onset of relaxation) used to fit the MDS results. The lowest temperature value was calculated directly from the mean squared displacement reported in ref 12 . Although it has an approximate linear $T$-dependence in the temperature range shown, extrapolation of the dashed line to lower temperature indicates that the vibrations are anharmonic.

a fast relaxational step above the glass transition temperature in methanol is different from fragile polymers and small molecular liquids. The behavior of $F(k, t)$ for methanol is similar to that of glycerol $\left(\mathrm{C}_{3} \mathrm{H}_{5}(\mathrm{OH})_{3}\right)$, ${ }^{6,17}$ a structurally analogous alcohol. A previous $\mathrm{CM}$ analysis ${ }^{17}$ of neutron scattering data for glycerol showed that $\beta$ of the slow $\alpha$-process is temperature dependent, increasing to a value of 0.70 , typical of a "nonfragile" liquid, at $T=363 \mathrm{~K}$ for $k=1.2 \AA^{-1}$.

The methanol data at $k=8 k_{\min }$ (where $k_{\min }, \sim 0.24 \AA^{-1}$, is the smallest wavenumber compatible with a periodic boundary condition in SK's simulation) and temperatures from 135 to $300 \mathrm{~K}$ were fitted using eqs $1-3$ of the CM, as shown in Figure 3 (solid lines). The $A_{\mathrm{k}}(T)$ determined from the fits, displayed in Figure 4, does not have strictly a linear temperature dependence. Although the deviation from the latter is not large in the temperature range shown, this does not imply that the vibrations are harmonic throughout all temperatures. In fact, an extrapolation of the dashed line to zero temperature yields an intercept for $\log A_{\mathrm{k}}(T)$ that is significantly larger than zero, indicating anharmonicity of the vibrations. Direct evidence of anharmonic behavior for methanol vibrations in the glassy phase has been found by Bermejo and co-workers ${ }^{28}$ from inelastic neutron scattering measurements. Our analysis of the SK data is not inconsistent with this finding. Also, any uncertainties in the $A_{\mathrm{k}}(T)$ used in our CM fits to the SK data change neither the conclusion that the fast $\alpha$-process is weak nor (significantly) the values of the relaxations parameters so determined. The same statements can be made regarding a previous CM analysis ${ }^{16}$ of a molecular dynamics simulation of $o$-terphenyl, a "fragile" liquid that has a smaller $\beta$ and a prominent fast $\alpha$-process. We have shown ${ }^{29}$ that uncertainties of the DebyeWaller factor within reasonable limits do not alter the conclusion that the contribution to $F_{\mathrm{s}}(k, t)$ from relaxation is describable as a crossover from fast noncooperative relaxation (eq 1) to slowed cooperative relaxation (eq 2 ) at a temperature-insensitive time.

The Kohlrausch exponent $\beta$ was found to increase with increasing temperatures; the respective values of $\beta$ and $\tau^{*}$ are 0.66 and $3000 \mathrm{ps}$ at $T=170 \mathrm{~K}, 0.68$ and $450 \mathrm{ps}$ at $180 \mathrm{~K}, 0.69$ and $20 \mathrm{ps}$ at $210 \mathrm{~K}, 0.8$ and $4.2 \mathrm{ps}$ at $250 \mathrm{~K}$, and 0.91 and 1.6 ps at $300 \mathrm{~K}$. The crossover from the fast $\alpha$-relaxation (simple exponential) to slow $\alpha$-relaxation (stretched exponential) in the vicinity of $t_{\mathrm{c}}=1.7 \mathrm{ps}$ occurs in the manner illustrated by curve $i$ in the inset of Figure 1. Clearly, the fast $\alpha$-relaxation is very weak at all temperatures. This is in contrast to this crossover as seen in the neutron scattering data for several polymers ${ }^{4,5}$ and in MDS data of $o$-terphenyl, ${ }^{16}$ all of which are fragile liquids exhibiting a relaxation similar to curve ii) in the Figure 1 inset.

The distinguishing feature of the $F(k, t)$ for methanol is the severe lack of prominence of the fast $\alpha$-relaxation for $T<210$ $\mathrm{K}$. This conclusion can be drawn by subtracting the two functions 1 and 2 at $t_{\mathrm{a}}=0.5 \mathrm{ps}$ in Figure 3 and comparing the difference with Figure 1. The amount of decay, $1-\exp \left(-t_{\mathrm{c}}\right)$ $\tau_{0}$ ), of the fast $\alpha$-relaxation at $t_{\mathrm{c}}$ is also negligibly small for methanol, as seen in Figure 2 in which the data points represented by filled circles correspond to the methanol $\beta$ and $\tau^{*}$ values from the $F_{\mathrm{S}}(k, t)$ data at 170,180 , and $210 \mathrm{~K}$. Not only does the fast $\alpha$-relaxation for methanol have a lack of prominence as in the case for glycerol ${ }^{17}$ but the former's relaxation times, $\tau^{*}$, as well as $\beta$, for the slow $\alpha$-process at 210 $\mathrm{K}$ are nearly equal to the values determined for glycerol at $T=$ $363 \mathrm{~K}$ and $k=1.2 \AA^{-1} .6,17$

The analysis herein is limited to data at fixed $k \approx 2 \AA^{-1}$, since $F_{\mathrm{SH}}(\mathrm{t})$ was only given in ref 12 for that value of the wavenumber. However, Sindzingre and Klein ${ }^{12}$ reported the variation of the mean relaxation time with $k$. There is a prediction of the coupling model, valid only when the Gaussian approximation holds, which can be used to corroborate the fits of eq 2 to the MDS data. The model predicts for the wavenumber dependence of the relaxation $\tau^{*}(k) \propto k^{-2 / \beta}$. Since $\beta$ is approaching unity at the highest temperatures in Figure 3, we expect a quadratic dependence of the relaxation time on $k$. Indeed, we find that at the highest temperatures, the relaxation times in ref 12 approach a $k^{-2}$ dependence. At lower temperatures, there is insufficient long-time decay of the $F_{\mathrm{SH}}(t)$ for us to check this prediction.

Molecular dynamics simulations depend on the choice of the intermolecular potential. As pointed out by SK, there are discrepancies between the results from their MDS and from the molecular dynamics of real methanol. The simulated methanol is too compressed, resulting in calculated values of compressibility, thermal expansion coefficient, and the translational diffusion coefficient $D$, which are all too small. The mobility of the simulated methanol is less than that experimentally found for real methanol ${ }^{30}$ and decreases more rapidly with decreasing temperature. We refer to this hypothetical glass-former as (methanol $)_{\mathrm{SK}}$, distinguishing it from actual methanol. We expect the former's overly compressed state to result in stronger 


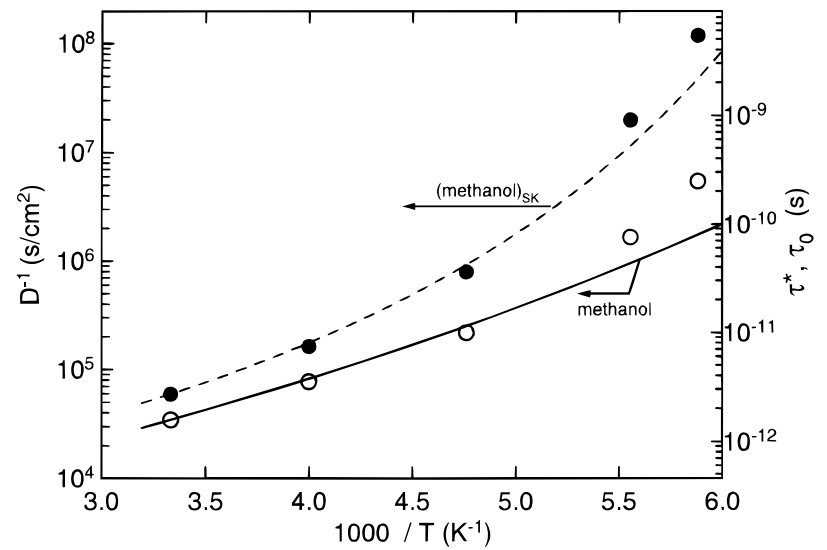

Figure 5. Respective Vogel-Fulcher fits of the inverse of the diffusion constant calculated by SK ${ }^{12}$ from their MDS of methanol (dashed line, from ref 12) and measured for real methanol (solid line, taken from ref 23), along with the noncooperative $(O)$ and intermolecularly cooperative $(\mathbf{0})$ relaxation times deduced from fitting the MDS data (see Figure 3 ). The $\tau^{*}$ values have been multiplied by 1.8 to bring them into coincidence with the highest temperature MDS results for $D^{-1}$, illustrating their similar temperature dependences.

intermolecular cooperativity (more "crowding coupling"), which should be manifested as a smaller $\beta$ and greater "fragility" than found for real methanol.

In Figure 5, the calculated (dashed curve) and the VogelFulcher fit to measured values of $D^{-1}$ are displayed, illustrating that the dynamics of real methanol are not reproduced by (methanol $)_{\text {SK. }}$. The temperature dependence of the relaxation times $\tau^{*}(k)$ and $\tau_{0}(k)$ deduced from our analysis (Figure 2) of (methanol) $)_{\mathrm{SK}}$ are also shown in Figure 5. The respective ordinate scales have been adjusted to bring the values of the cooperative relaxation time, $\tau^{*}(k)$, into coincidence with the simulation results for $D^{-1}$. The temperature dependencies are comparable, which is unsurprising, since the $F_{\mathrm{S}}(k, t)$ and the mean square displacement are related. ${ }^{31}$

More interestingly, the temperature dependence of $\tau_{0}(k)$ is close to that of $D^{-1}$ measured for real methanol, ${ }^{30}$ the latter varying less with temperature than $D^{-1}$ for (methanol) SK. This $_{\text {S }}$ indicates that real methanol is a "stronger" liquid with possibly a larger $\beta$ than the simulated methanol of ref 12 . Dielectric measurements ${ }^{32}$ have indicted that the loss peak for methanol has very nearly the Debye form (corresponding to an exponential correlation function) in the sense that its full width at half height of the dielectric loss peak is only a factor of 1.02 wider over the entire temperature region of Figure 5. The more recent data of ref 32 is consistent with earlier reported data in the frequency range $0.95-89 \mathrm{GHz}$ by Barthel et al. ${ }^{33}$ The dielectric relaxation data depart from the Debye form starting approximately at frequencies higher than the loss frequency by a factor of 10 , and this is taken as evidence of the existence of two additional dielectric relaxation processes at high frequencies. The existence of three distinct relaxation process in the dielectric spectrum was also found in MDS by Skaf et al. ${ }^{34}$ and in the dielectric spectrum calculated from simulated data by Bermejo et al. ${ }^{35}$ The origins of the three dielectric processes have been elucidated by MDS. ${ }^{34}$ Among these three processes, the Debye relaxation has the largest dielectric strength, more than an order of magnitude larger than the others, irrespective of temperature. ${ }^{33}$ Naturally, the nearly Debye process is identified with the slow $\alpha$-relaxation of real methanol, with a $\beta$ effectively equal to 1 . In this circumstance, the CM predicts the fast $\alpha$-relaxation to be either absent or very weak, notwithstanding the presence in the experimental dielectric spectrum of higher frequency loss processes. It should be mentioned that the dielectric relaxation measurements of Barthel et al. carried up to about $300 \mathrm{GHz}$ (corresponding to $t=0.5 \mathrm{ps}$ ) show only one peak, monotonically decreasing toward higher frequency. The susceptibility minimum predicted by $\mathrm{MCT},{ }^{13}$ and seen in dielectric data of glycerol $^{36}$ and $\mathrm{CKN},{ }^{18,37}$ is not observed.

Although the $\beta$ for dielectric relaxation of real methanol is

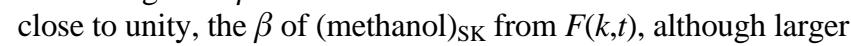
than for fragile liquids, has a value significantly less than 1 , particularly at lower temperature. This difference between the respective $\beta$ 's of (methanol) SK $_{\text {and real methanol is consistent }}$ with the differing temperature dependencies of their respective $D^{-1}$ and $\tau^{*}(k)$. Moreover, the nearly Debye-like behavior of real methanol as probed dielectrically, implying weak dynamic constraints therein, is the reason that the temperature dependence of the noncooperative relaxation time, $\tau_{0}(k)$ from eq 3 , approximates that of the $D^{-1}$ of real methanol. ${ }^{23}$ All these results are consistent with an "overly compressed state" in the MDS calculation. We expect the fast $\alpha$-process in real methanol would be even weaker than in (methanol) SK $_{\mathrm{SK}}$ and the $T$ dependence of $\tau^{*}$ for real methanol in better agreement with the measured $D^{-1}$. However, at this time, the available experimental results on methanol ${ }^{38,39}$ do not have the detail necessary for a comparison with the $F_{\mathrm{SH}}(t)$ data ${ }^{12}$ analyzed herein.

\section{Summary}

Glass-forming liquids and polymers divide themselves into two categories based on how their relaxation behavior responds to temperature changes. Experimentally, only for fragile liquids is the short-time dynamics associated with the existence of a prominent fast process (i.e., the CM's fast $\alpha$-relaxation or the fast $\beta$-process of MCT). The coupling model offers an explanation of this correlation between the fast dynamics and fragility. A prominent fast $\alpha$-process is expected in fragile liquids, such as (most) polymers, and fragile nonpolymeric glassformers, such as $0.6 \mathrm{Ca}\left(\mathrm{NO}_{3}\right)_{2}-0.4 \mathrm{KNO}_{3}$ and $o$-terphenyl, all of which have smaller $\beta$. Conversely, weak, fast $\alpha$-processes are predicted in strong and intermediate liquids, including $\mathrm{ZnCl}_{2}$, $\mathrm{B}_{2} \mathrm{O}_{3}$, glycerol, and methanol. Analysis of the molecular dynamics simulation data of methanol has confirmed that the fast $\alpha$-process at picosecond time scales is weak in this strong liquid. The slow $\alpha$-process has large $\beta$, as expected for strong glass-forming liquids. ${ }^{24-27}$ These conclusions are supported by dielectric relaxation data. The respective temperature dependencies found for the fast and the slow $\alpha$-processes elucidate the difference in intermolecular interactions between simulated and real methanol.

Acknowledgment. This work was supported by the Office of Naval Research.

\section{References and Notes}

(1) Knaak, W.; Mezei, F.; Farago, B. Europhys. Lett. 1988, 7, 527.

(2) Mezei, F. Ber. Bunsen-Ges. Phys. Chem. 1991, 95, 1118.

(3) Petry, W.; Wuttke, J. Transp. Theory Stat. Phys. 1995, 24, 1075. 2603.

(4) Colmenero, J.; Arbe, A.; Alegria, A. Phys. Rev. Lett. 1993, 71,

(5) Zorn, R.; Arbe, A.; Colmenero, J.; Frick, B.; Richter, D.; Buchenau, U. Phys. Rev. E 1995, 52, 781.

(6) Wuttke, J.; Petry, W.; Coddens, G.; Fujara, F. Phys. Rev. E 1995, $52,4026$.

(7) Cummins, H. Z.; Li, G.; Du, W. M.; Hernandez, J.; Tao, N. J. Transp. Theory Stat. Phys. 1995, 24, 981.

(8) Wuttke, J.; Hernandez, J.; Li, G.; Coddens, G.; Cummins, H. Z.; Fujara, F.; Petry, W.; Sillescu, H. Phys. Rev. Lett. 1994, 72, 3052.

(9) Steffen, W.; Patkowski, A.; Glazer, H.; Meier, G.; Fischer, E. W. Phys. Rev. E 1994, 49, 2992.

(10) Lewis, L. J.; Wahnström, G. Phys. Rev. E 1994, 50, 3865. 
(11) Signorini, G. F.; Barrat, J.-L.; Klein, M. L. J. Chem. Phys. 1990, 92, 1294.

(12) Sindzingre, P.; Klein, M. J. Chem. Phys. 1992, 96, 4681.

(13) Götze, W.; Sjögren, L. Rep. Prog. Phys. 1992, 55, 241. 52 .

(14) Zorn, R.; Richter, D.; Frick, B.; Farago, B. Physica A 1993, 201,

(15) Roland, C. M.; Ngai, K. L. J. Chem. Phys. 1995, 103, 1152.

(16) Roland, C. M.; Ngai, K. L.; Lewis, L. J. J. Chem. Phys. 1995, $103,4632$.

(17) Ngai, K. L.; Roland, C. M. J. Chem. Phys. 1997, 106, 1187.

(18) Ngai, K. L.; Cramer, C.; Satkamp, T.; Funke, K. In Nonequilibrium Phenomena in Supercooled Fluids, Glasses and Amorphous Materials; Giordano, M., Leporini, D., Tosi, M. P., Eds.; World Scientific: Singapore, 1996; p 3.

(19) Angell, C. A. In Relaxations in Complex Systems; Ngai, K. L., Wright, G. B., Eds.; Government Printing House: Washington, DC, 1995; p 3. Available on request to the editors.

(20) Ngai, K. L. Comments Solid State Phys. 1979, 9, 127. Ngai, K. L.; Tsang, K.-Y. Macromol. Symp. 1995, 90, 95. Ngai , K. L.; Peng, S. L.; Tsang, K.-Y. Physica A 1992, 191, 523. Ngai, K. L.; Rendell, R. W. J. Non-Cryst. Solids 1991, 233, 131. Rendell, R. W. Phys. Rev. E 1993, 48, R17. Tsang, K.-Y.; Ngai, K. L. Phys. Rev. E 1996, 54, R3067. For a review of applications of the CM, see the following. Ngai, K. L. In Disorder Effects on Relaxational Processes; Richert, R., Blumen, A., Eds.; Springer-VerlagL Berlin, 1994; p 89.

(21) Lebon, M. J.; Dreyfus, C.; Li, G.; Aouadi, A.; Cummins, H. Z.; Pick, R. M. Phys. Rev. E 1995, 51, 4537. See comments on this work in Ngai, K. L.; Roland, C. M. Phys. Rev. E 1996, 54, 6969.

(22) Brodin, A.; Engberg, D.; Torell, L. M.; Börjesson, L.; Sokolov, A. P. Phys. Rev. B 1996, 53, 11511.
(23) Angell, C. A. Science 1995, 267, 1924.

(24) Plazek, D. J.; Ngai, K. L. Macromolecules 1991, 24, 1222.

(25) Böhmer, R.; Ngai, K. L.; Plazek, D. J.; Angell, C. A. J. Chem Phys. 1993, 99, 4201.

(26) Roland, C. M.; Ngai, K. L. Macromolecules 1991, 24, 5315; 1992 $25,1844$.

(27) Roland, C. M.; Ngai, K. L. Macromolecules 1996, 29, 5747.

(28) Bermejo, F. J.; Alonso, J.; Criado, A.; Mompean, F. J.; Martinez, J. L. Phys. Rev. B 1992, 46, 6173.

(29) Roland, C. M.; Ngai, K. L. Mater. Res. Soc. Proc. Series, in press.

(30) Karger, N.; Vardag, T.; Ludemann, H. D. J. Chem. Phys. 1990, 93, 3437.

(31) Roland, C. M.; Ngai, K. L. J. Chem. Phys. 1996, 104, 2967.

(32) Stickel, F. J. Ph.D. Thesis, Fachbereich Physik der Johannes Gutenberg Universität Mainz, 1995.

(33) Barthel, J.; Bachhuber, K.; Buchner, R.; Hetzenauer, H. Chem. Phys. Lett. 1990, 165, 369.

(34) Skaf, M.; Fonseca, T.; Ladanyi, B. M. J. Chem. Phys. 1993, 98, 8929.

(35) Bermejo, F. J.; Enciso, E.; Garcia-Hernadez, M.; Martinez, J. L.; Fak, B.; Mason, T. J. Non-Cryst. Solids 1994, 167, 172

(36) Lunkenheimer, P.; Pimenov, A.; Dressel, M.; Goncharov, Yu. G.; Böhmer, R.; Loidl, A. Phys. Rev. Lett. 1996, 77, 318.

(37) Lunkenheimer, P.; et al. Europhys. Lett. 1996, 33, 611.

(38) Bermejo, F. J.; Batallan, F.; Encisco, E.; White, R.; Dianoux, A. J.; Howells, W. S. J. Phys.: Condens. Matter 1990, 2, 1301.

(39) Bermejo, F. J.; Batallan, F.; Howells, W. S.; Carlile, C. J.; Enciso, E.; Garcia-Hernandez, M.; Alvarez, M.; Alonso, J. J. Phys. Condens. Matter 1990, 2, 5005 